In Cres. Vol. $3 N^{\circ}$ 2: pp. 299-311, 2012

\title{
LA FAMILIA Y LAS REDES SOCIALES EN LOS TRASTORNOS ALIMENTICIOS EN ADOLESCENTES DE LA ÉPOCA CONTEMPORÁNEA*
}

THE FAMILY AND THE SOCIAL NETWORKS IN THE FOOD DISORDERS IN TEENAGERS OF THE CONTEMPORARY EPOCH

\section{Zelmira Beatriz Lozano Sánchez ${ }^{1}$}

\section{RESUMEN}

En el presente trabajo se analiza la influencia negativa que ejercen las redes sociales en los transtornos alimenticios, especialmente la anorexia y la bulimia en los jóvenes adolescentes de la sociedad actual. Por lo tanto, es importante que las familias y la educación adopten medidas preventivas y de promoción de la salud mental, en resguardo del desarrollo sano de la juventud.

PALABRAS ClAVE: Transtornos alimenticios, Anorexia, Bulimia, Redes sociales.

\begin{abstract}
In the present work there is analyzed the negative influence that the social networks exercise in the food transtornos, specially the anorexia and the bulimia in the teen young persons of the current company. Therefore, it is important that the families and the education adopt preventive measures and of promotion of the mental health, in security of the healthy development of the youth.
\end{abstract}

KEY WORDS: Food disorders, Anorexia, Bulimia, Social networks.

* Recibido: 04 de mayo del 2012; aprobado: 25 de octubre del 2012.

1 Psicóloga. Mag. Docencia Universitaria e Investigación Pedagógica. Profesora en la Universidad Privada Antenor Orrego y en la Escuela de Postgrado de la Universidad César Vallejo. 


\section{INTRODUCCIÓN}

En palabras de Unikel (1999) "Los trastornos alimenticios son un grave problema de salud pública que afecta, por lo general, a un gran número de mujeres en las sociedades occidentales. Actualmente existen características actitudinales y conductuales hacia el peso, la figura y el comer comunes en la mayoría de las mujeres, o normativas dentro de nuestro contexto sociocultural, como el uso de dietas para bajar de peso, insatisfacción con la figura, el peso y el deseo de pesar menos. La práctica de algunas de estas conductas puede ser el detonador de una patología más grave. Estos trastornos son enfermedades que se desarrollan principalmente en la adolescencia o en mujeres jóvenes de edades entre los 12 y 25 años el 90-95\% de los casos son mujeres".

Ahora bien, cabe destacar que no es la moda la que influye en la belleza, sino el prototipo de las modelos; anteriormente en los años 45 y 50, la mujer rellena era bien vista; a partir de los 60 aproximadamente son las mujeres delgadas quienes llevan la primacía del modelo. Ya que en los concursos de belleza imponen que así deben ser las mujeres bellas.

Según Edelstein (1999): "La delgadez se ha convertido para algunas mujeres, cada vez más jóvenes, en una especie de culto del que no pueden prescindir. Sacrifican horas enteras dedicadas al ejercicio físico para conseguir adelgazar. Se someten a privaciones con dietas difíciles de cumplir y terminan desarrollando una verdadera obsesión por estar delgadas que, en algunos casos, da lugar a una enfermedad por dependencia. Eso ocurre con las anoréxicas, que acaban deificando la delgadez, haciéndose dependientes del símbolo de ésta, por lo que pueden llegar hasta morir".

La adolescencia representa uno de los grupos más vulnerables para el desarrollo de trastornos de la alimentación más común en mujeres, quienes modifican su alimentación persiguiendo el "ideal de belleza" impuesto por la cultura occidental. La presión social por estar delgado contribuye a que los adolescentes acepten o rechacen su imagen corporal, comiencen con alguna dieta para perder peso llevándola sin control y supervisión adecuada (O'connor, 1997).

Las tasas de prevalencia de los trastornos alimenticios son más altas en adolescentes en edad universitaria que en estudiantes de la escuela secundaria (Gómez, G. 1993), este dato se corrobora con la población de adolescentes del presente estudio, pues se observa un incremento significativo en este grupo poblacional.

Se considera que la presencia de estos trastornos alimenticios se ven acelerados por la actual dinámica familiar de los adolescentes, ya que estos trastor- 
nos se presentan principalmente durante la adolescencia. La familia debe implicarse en la detección del problema, en el tratamiento y, por supuesto, representa el fundamental agente preventivo.

La familia, como grupo natural, elabora en el curso del tiempo pautas de interacción que constituyen su estructura, la cual rige el funcionamiento de sus miembros, facilita la interacción recíproca y define una gama de conductas posibles. Sin embargo, la realidad actual señala que la dinámica de las familias ha cambiado, pues predominan las familias disfuncionales y desintegradas, en la que los padres ya no dirigen los patrones comportamentales de sus hijos, por lo que la conducta de estos está definida por otros estereotipos que no se ajustan a su realidad y obedecen a características de otros lugares, surgiendo de esta manera la conducta "alienada". Los hijos actúan influenciados por los medios y el grupo de pares, adoptando las conductas dictadas desde esos ámbitos(Rodríguez Sacristán, J.1995).

Se dice que toda la familia se desplaza de manera natural hacia un sistema más individualizado y diferenciado, lo que trae consigo alta inestabilidad de las reglas familiares, aumenta la ansiedad y los conflictos. Suelen ocurrir grandes cambios en el estilo de vida: retiro laboral, divorcio, nuevas nupcias, cambios de empleo, etc. Para el adolescente es un duelo el ir abandonando la seguridad de la dependencia infantil, así como el quiebre de la imagen parental idealizada (Bruch, H. 1971).

A nuestro parecer, si desde la familia, se le brinda al adolescente seguridad, afecto, respeto, límites y verdaderos modelos, él encontrará las bases para su desarrollo, influyendo en el logro de una personalidad integrada, dándole pertenencia a una familia. Por tanto el papel de la familia en este terreno es decisivo, aun cuando la sociedad intente imponer normas absolutamente contrarias.

Por otro lado, tenemos la presencia de los medios de comunicación y las tecnologías de la información. Cuanto mayor es la exposición a estos contenidos mayor es el riesgo de padecer trastornos Cuanto más tiempo pasan las chicas adolescentes participando en redes sociales, más posibilidades tienen de desarrollar una imagen negativa de su propio cuerpo, así como diversos trastornos de la alimentación, como la anorexia o la bulimia. En la actualidad, las redes sociales juegan un papel muy importante en el incremento de la anorexia y la bulimia de los jóvenes. Cabe recalcar que los adolescentes son más propensos a ser influenciados rápidamente por las cosas que leen o ven por internet, ya que debido a su edad son demasiado volubles. Estas redes sociales son de fácil acce- 
so y disponibilidad y cubren todos los espacios donde el adolescente interactúa, característica que le resulta atractiva por la gran capacidad de persuasión y convencimiento, en donde también la información que requieren es muy rápida. Estas redes se convierten en vehículos eficaces para transmitir y adoptar determinados estereotipos. Creo que este es el principal riesgo, ya que estas redes remplazan a la familia, sobre todo a los roles y funciones en la formación de los hijos.

Todo lo mencionado afecta y repercute en el desarrollo de la persona, por lo que es necesario plantear una educación que garantice su formación y desarrollo integral, en tal medida que asegure el desarrollo social y nacional. Consideramos que para que la educación del hombre sea una realidad también es una responsabilidad política del estado, pues hay que reconocer que es la persona el primer sujeto que debe ser protegido.

\section{LOS TRASTORNOS ALIMENTICIOS}

Los trastornos alimentarios son alteraciones de las conductas relacionadas con la ingesta como consecuencia de los dramáticos esfuerzos por controlar el peso y la silueta. La característica común de todos los trastornos alimentarios son los comportamientos anormales de alimentación. Son problemas serios de salud mental y pueden poner en peligro la vida. (Lugli Z, Vivas E, 2001). La frecuencia de estos trastornos es más elevada en el sexo femenino, y suele estar generados por la combinación de factores individuales y familiares, junto a la presión cultural por mantenerse delgadas.

A nivel psicopatológico, y siguiendo la cuarta edición del Manual Diagnóstico y Estadístico de Trastornos Mentales y del Comportamiento (DSM), de la Asociación Psiquiátrica Americana, dentro de los trastornos de la conducta alimentaria, se distingue a la anorexia nerviosa y la bulimia nerviosa. En este trabajo se consideran estos dos trastornos, dado el aumento significativo de incidencia y prevalencia de las mismas en los últimos años.

La característica esencial de la anorexia consiste en el rechazo a mantener el peso corporal mínimo normal, en un miedo intenso a ganar peso y en una alteración significativa de la percepción de la forma o tamaño del cuerpo (American Psychiatric Association APA, 2002). La pérdida de peso puede deberse tanto a una dieta muy restrictiva o la realización de ejercicio físico muy intenso, como a las purgas.

En la anorexia nerviosa, lo que la define no es una auténtica pérdida o falta de apetito, sino el deseo irrefrenable de alcanzar la delgadez a toda costa 
(Rausch C, Bay L, 1997). Es habitual que esta alteración se inicie por recibir alguna crítica directa o indirecta en relación a su peso (Bruch, H. 1971). Los investigadores han descubierto que los anoréxicos en realidad sienten hambre. Experimentan sensaciones cognoscitivas de hambre, junto con una fuerte preocupación por la comida. El paciente anoréxico experimenta un intenso miedo al aumento de peso a pesar de que éste disminuye cada vez más y de una manera alarmante. Se produce una distorsión de la imagen corporal, lo que obliga a mantener la dieta. También se dan síntomas de ansiedad, depresión y obsesivos. Esta malnutrición también provoca tristeza, irritabilidad, aislamiento social e incluso ideas de suicidio.(Saldaña C. 2000).

Entonces podemos decir que las tres áreas principales de funcionamiento psicológico alterado en los anoréxicos son: la imagen corporal; la interpretación de los estímulos internos y externos, sobre todo del hambre; y un sentimiento abrumador de ineficacia, tal como lo sustenta Caparros N y Sanfeliú I. (1997).

Otro de los trastornos alimenticios que se considera es la bulimia, que viene a ser una ingestión episódica, desenfrenada y rápida de grandes cantidades de comida en un tiempo corto (atracón), produciendo sentimientos de malestar físico y emocional. No hay pérdida de peso. (Barlow, 2001).

Se caracteriza por episodios cíclicos y recurrentes de voracidad (atracones) seguidos por conductas compensatorias inadecuadas como el vómito autoinducido, el abuso de laxantes y diuréticos, el ayuno drástico y/o el ejercicio excesivos. La paciente está consciente de que su patrón alimentario no es normal, que escapa de su control, por lo que se angustia enormemente y siente posteriormente gran culpa, autodesprecio, vergüenza y depresión. Todo esto los torna muy ansiosos, sensibles en el ámbito interpersonal y propenso a somatizaciones, pudiendo llegar a trastornos del estado de ánimo y de la ansiedad, e inclusive todo esto les puede llevar al suicidio.(American Psychiatric Association [APA], 2002).

Podemos decir que estos trastornos presentan una multicausalidad, pues intervienen los aspectos biológicos, psicológicos y sociales, con un predominio, a nuestro parecer de los aspectos familiares y socio-culturales.

Sin duda, la preocupación y sobrevaloración de una sociedad que engorda por su dieta y estilo de vida, va a determinar que los problemas en la autovaloración y autoestima y las angustias y dependencias no resueltas del adolescente, con el consecuente estado depresivo y la alteración del apetito y la ingestión conduzcan a un deseo de delgadez, centrándose en una dinámica en la cual se pierde el control y se queda atrapado. 
Tal es así que, para alguien con un trastorno alimentario, la imagen corporal está íntimamente ligada a la auto-imagen. Pensamientos positivos acerca de su cuerpo la llevarán a pensamientos positivos acerca de ella misma. Si se siente "delgada" o "más delgada en un día en particular, pensará mejor de ella misma. Viceversa, si se siente "gorda" estará convencida de que es una mala persona.

Las víctimas de la anorexia y de la bulimia poseen un carácter obsesivo que acaba traduciéndose en una preocupación constante por el peso y la dieta. Sin embargo, la personalidad de estos pacientes es diferente. Así, la anoréxica suele estar considerada como "niña modelo": perfeccionista, buena estudiante, con un nivel intelectual alto y con tendencia a evitar conflictos. En cambio, su preocupación por la opinión que los demás tienen de ella es excesiva, así como su autocontrol. Las bulímicas, por el contrario, suelen ser más impulsivas, intolerantes y se frustran más. (Jhonson, B. 2000). Estamos de acuerdo con este planteamiento, ya que estas características se han podido observar con mucha incidencia en las estudiantes universitarias.

Pensamos que más allá de las consecuencias físicas de estos trastornos, la huella emocional y psicológica es la que trasciende la vida de las adolescentes, ya que el cargo de conciencia de haber hecho sufrir a su familia y/o haber dejado los estudios o trabajo para recuperase, etc. es bastante significativo y profundo, sin dejar de lado que una vez que la persona se recupera podría sentir que su integración en la sociedad será difícil.

\section{FAMILIA Y TRASTORNOS DE LA CONDUCTA ALIMENTARIA}

Una atención significativa se le ha prestado a las dinámicas familiares de las personas con trastornos alimentarios. Según estudios de investigación (Cook-Darzens et al, 2005) realizados a las familias y adolescentes con trastornos alimenticios, han reportado datos importantes, tales como:

- La calidad del funcionamiento familiar juega un rol importante en el curso y resultado del trastorno.

- Los sujetos con trastornos alimentarios perciben a sus familias como significativamente menos adaptables, menos cohesionadas y más pobremente comunicativas, comparadas con personas control sin trastornos alimentarios.

- En cuanto al ambiente familiar de estudiantes universitarios se encontró que, en la medida que la disfunción familiar se incrementa, aumenta también el reporte de trastornos de conducta alimentaria. 
Tomando como referencia estos hallazgos, se podría decir que los trastornos de la conducta alimentaria podrían ser entendidos como un síntoma de la disfunción familiar. En algunos casos falta calidad a los vínculos entre los miembros de la familia. En lugar de que sean flexibles, respetuosos y balanceados, donde cada persona involucrada sienta la liberad de ser él o ella misma, las relaciones tienden a caer en tres categorías: demasiado cercanas (atrapados), demasiado distantes (desconectados) o una errática combinación de las dos. (White, 2011).

Podemos recalcar que hay ciertas características particulares, tanto en las familias de anoréxicos como bulímicos. En los anoréxicos podemos encontrar familias aglutinadas que dificultan el proceso de separación individuación de los hijos. Familia unida y sobreadaptada a las reglas sociales, interesada en la eficacia y el buen rendimiento de sus hijos, son familias que buscan y valoran el éxito. Afectos desvalorizados, deficiencia en los vínculos primarios. Madre con poca capacidad empática hacia sus hijos, dificultad para decodificar las necesidades de los hijos, sobrevaloración de lo estético y lo intelectual. El padre generalmente está presente narcisistamente, con gran sentimiento de autoorgullo. Por lo general son pacientes introvertidos, tienen pocos amigos, muy apoyados en la madre y son descritos por ésta como los hijos ideales (Minuchín y col., 1978). Esto concuerda con lo que sostiene White, al describir a la familia de la anoréxica como un sistema rígido de creencias implícitas que se transmiten de generación en generación. "Son familias con graves dificultades para la individuación y para la solución de problemas”. En 1996, el propio White añade a lo anterior la influencia social y destaca el estereotipo de los sexos en una sociedad patriarcal. Señala al respecto que para que una mujer sea valorada debe mostrarse dependiente y realizarse emocionalmente cuidando a los demás. Para sentirse digna y valiosa debe conseguir el ideal de delgadez.

Strober y Yager (2012) describen dos modelos familiares típicos de las familias de pacientes anoréxicas. Habría "familias centrípetas", en las que hay una excesiva cohesión, falta de permisividad, poca expresión emocional y pobres contactos extrafamiliares y "familias centrífugas", poco cohesionadas, altamente conflictivas, con miedo al abandono, excesiva dependencia, falta de control y conflicto de pareja.

Se comparte la posición de Garfinkel y Garner (2012), al criticar los modelos anteriores por alejarse de la realidad clínica y plantear que las familias, en los TCA, son heterogéneas, no existiendo una "tipología familiar". En su lugar, hablan de una serie de factores de riesgo y dificultades familiares que 
actuarían como elementos predisponentes. En los últimos años es la teoría más aceptada.

Por otro lado, las familias de los bulímicos son aglutinadas y dificultan el proceso de separación individuación de los hijos; suelen ser padres divorciados o ausentes. Los roles familiares están alterados, madres muy dominantes y directivas, sobreprotectoras. En este tipo de familia los afectos son conflictivos. Los padres suelen ser inmaduros e impulsivos. Se encuentran antecedentes de familiares con sindromes distímicos. Los sujetos con bulimia nerviosa son personas extrovertidas, poco voluntariosas, son conflictivos, muestran necesidad de realizar cosas para llamar la atención de los padres (alcohol, robo, drogas, mentiras, etc.).Visto así, las familias de bulímicas son las más patológicas, apareciendo hostilidad, defectos nutricionales, desligamiento, impulsividad y falta de empatía parental. No suele aparecer conflicto conyugal. (Baravalle G y Vacarreza L., 1998).

Cabe señalar que la sociedad actual plantea otras necesidades y retos. En este sentido, las familias tienen nuevos ideales y, por ende, nuevas formas y vías de satisfaserlos, ocupando la mayor parte de su tiempo en ello, dentro y fuera del medio familiar. Esto ocasiona un abandono y descuido familiar, tanto en la educación como en la formación de sus hijos. Por otro lado, los hijos adolescentes que están en búsqueda de su identidad, de su imagen personal y autoconcepto, necesitan ser atendidos, necesitan darle un sentido a su vida y elaborar un proyecto de vida; pero esa búsqueda lo encontrarán en los amigos, en las redes sociales, que le servirán de modelos, que no siempre son los más adecuados y positivos para su vida. Y es en la adolescencia donde aparecen las primeras preocupaciones por la figura que, en ocasiones, se traducen en comportamientos nocivos para la salud que pueden desembocar en enfermedades como la anorexia y la bulimia.

\section{LAS REDES SOCIALES Y LOS TRASTORNOS ALIMENTICIOS}

Las redes sociales, como lo dice su nombre, son para cumplir finalidades sociales. A pesar de que esta herramienta puede ser usada a cualquier edad las estadísticas indican que más de la mitad de usuarios son jóvenes y adolescentes (el 80 por ciento tiene entre 12 y 30 años). Además, en estos lugares se crea algo muy importante, llamada "la lista de amigos", para los jóvenes y adolescentes amigos en las redes sociales no tiene el mismo significado que en la vida real. A principios de la década del 2000 aparecen más sitios web que permiten crear un círculo de amigos virtuales, compartir datos personales e información. A partir del 2003 se fue popularizando y se crearon más sitios como los primeros. 
"En la actualidad existen más de 200 redes sociales, con más de 800 millones de usuarios en todo el mundo. Una tendencia que crece cada mes". (Morduchowicz, Marcon, Sylvestre y Ballestrini, 2010, p. 6).

En el mundo de hoy, encontramos miles de redes sociales para todo tipo de gusto. La mayoría de jóvenes solo buscan divertirse jugando y pasar un buen rato navegando en internet. Por otro lado, buscan también construir un sitio personal, en el cual puedan compartir experiencias y averiguar los últimos sucesos y novedades.

Se ha hecho más relevante la cantidad de amigos que la calidad. Los adolescentes se preocupan por cada uno de estos amigos y sus opiniones. Todo esto lleva a que uno se pregunte porqué son tan importantes las redes sociales entre los jóvenes que hace que atribuyan un gran porcentaje de su tiempo a ello. Ya que la edad promedio que usa esta herramienta son jóvenes pues uno de sus principales propósitos es formar una personalidad definida que los demás acepten, o ya tener una personalidad y quieren compartirla con otros para comprobar el grado de aceptación que tienen. Es allí donde entra la lista de amigos, ya que es esa lista la que va a juzgar, criticar o alabar según sea el caso. En las redes sociales, "amigo" es todo aquel que ha sido invitado a visitar el sitio personal en la red". (Morduchowicz et al., 2010, p. 3).

A pesar de que la actual generación vive rodeada de tecnología, sus amistades siguen siendo tan importantes como en generaciones pasadas, sólo que ahora existen diferentes mecanismos de comunicación entre ellos.

Las investigaciones demuestran que cuanto mayor es la exposición de las jóvenes a contenidos de moda a través de Internet y a ciertos programas de televisión, también es mayor la posibilidad de que las niñas desarrollen anorexia y otros trastornos alimenticios.

Por otro lado, los adolescentes suelen tomar muy en cuenta las opiniones de sus amigos y familiares. Es por ello que estos deben evitar dar comentarios negativos o mofarse acerca del cuerpo de un adolescente, pues esto podría traer como consecuencia el inicio de un trastorno alimenticio. Asimismo, las adolescentes en comparación con los chicos, son las que más se encuentran propensas a caer en las enfermedades de la anorexia y la bulimia, pues ellas se ven más influenciadas por información en internet acerca de artistas, modelos, actrices etc., lo cual la mayoría de veces genera una idea errónea del cuerpo ideal, induciéndolas a perder peso y generando en ellas algún otro trastorno alimenticios.

Hay sitios en Internet que tienen como finalidad estrechar las redes sociales de sus usuarios, generalmente mujeres y niñas de entre 12 y 25 años, ofre- 
ciendo material escrito, imágenes, audio y aún videos cuyo contenido apoya actitudes y comportamientos encaminados a lograr un peso corporal excesivamente bajo. No sólo promueven prácticas alimenticias nada sanas, sino que también alientan conductas autodestructivas y aún suicidas. Las redes sociales virtuales -Facebook, Twitter, Tuenti y Delicious, entre las más populares-, acogen grupos que reivindican los desórdenes alimentarios como formas de vida, por lo que una vez más surge la advertencia de que hay que tener más cuidado con lo que aparece en la red, ya que existen portales de fácil acceso que alientan los desórdenes alimenticios entre adolescentes y jóvenes.(Méndez A. Carlos E. 1995).

El informe anual sobre la difusión y proliferación de contenidos de apología de la anorexia y la bulimia en la red (2010) pretende llamar la atención para que "el conjunto de organizaciones implicadas tomen medidas concretas para tratar de poner freno a este aumento de contenidos nocivos, altamente perjudiciales para la salud de los menores". Algunos datos que resalta el informe son los siguientes:

- Incremento de los sitios web que hacen apología de la anorexia y la bulimia: en un buscador como Google aparecen alrededor de 500.000 páginas pro anorexia y pro bulimia en menos de un segundo.

- Peligro de que este tipo de páginas puede conllevar a la sociedad y a la salud de las personas: el $75 \%$ de personas que consultan estos contenidos de la red son menores de edad.

- La apología de la anorexia y la bulimia está proliferando considerablemente a través de las redes sociales.

- Perfil de los usuarios: preferentemente chicas (95\%) que desean adelgazar a cualquier precio y se acercan de manera muy peligrosa a este tipo de trastornos.

Es así que estos trastornos alimenticios, anorexia y bulimia, tienen características diferenciales que permiten que los adolescentes armen grupos de pertenencia generados concretamente por la enfermedad y exponen el transcurso de la misma de forma abierta y pública, sin tener conciencia de ella. Lo dramático del asunto es que les ayudan a reafirmar su trastorno haciéndoles sentir que no están solos y que hay alguien en el mundo que los entiende (Gáfaro, Alejandra, 2001).

En este contexto, los padres juegan un papel fundamental. Estudios que se han hecho revelan que las hijas de padres pendientes del uso que éstas dan a los medios, de lo que sus hijas ven, o dispuestos a discutir con ellas los contenidos, 
presentan una mayor fortaleza personal que ejerce de protección contra este tipo de trastornos. (Méndez A. Carlos E. 1995).

Pienso que lo más importante es que los padres tengan conciencia. Deben regular el acceso a internet y saber a qué sitios se conectan sus hijos, porque es sumamente peligroso. Internet es un instrumento importantísimo de comunicación y, a la vez, un arma de doble filo.

Consideramos también que la mejor manera de evitar en los hijos trastornos alimenticios es fomentarles una actitud crítica sobre lo que hace a una persona mantenerse en el peso adecuado llevando una dieta equilibrada y realizando ejercicio físico, y distinguir el caso de aquellos que se esfuerzan en perder peso dañando a su organismo.

\section{CONCLUSIONES}

1. Todas las personas diseñan su identidad y al tratar de materializarla, dan forma a los rasgos de su personalidad. Para lograr esta identidad parten de diferentes modelos, algunos proceden de la familia y lo reciben como herencia cultural; otros utilizan diversos medios, generalmente con los que pasan gran parte de su tiempo. Los adolescentes que frecuentemente utilizan otros medios para diseñar su imagen personal, consideran que su mundo se reduce a su apariencia física y, por tanto, de ella depende su éxito social y personal. Las manifestaciones de este modo de entender y dar forma a su cuerpo están visibles en la sociedad del joven y reflejados en todos los grandes medios de comunicación.

2. Los estereotipos de "mujer ideal" que se observan en los medios de comunicación son productos construidos y fabricados por las empresas que las promocionan, influyendo de esta manera en la determinación del "modelo ideal". En tal sentido, surgen algunas conductas relacionadas a la preocupación por adelgazar, la insatisfacción con la imagen corporal y la práctica de dietas continuas, convirtiéndose en variables importantes en el estudio de los desórdenes de la conducta alimentaria y constituyendo un común denominador de las mismas, es decir, se presentan tanto en la anorexia nerviosa como en la bulimia nerviosa, presentándose predominantemente en mujeres adolescentes y jóvenes.

3. Las redes sociales se han convertido en aparentes familias de los cibernautas. El liderazgo lo ejerce el que desarrolló mejores y mayores destrezas para utilizar el medio. Por lo tanto, sus propuestas y opiniones son asimiladas y 
asumidas por quiénes se convierten en seguidores del líder. Así se inician y generalizan diferentes patrones de conductas, buenas y malas.

4. Los padres de familia con sus formas tradicionales de relacionarse con los miembros de su familia, no pueden competir con la capacidad provocadora y convencedora de los medios de comunicación, razón por la cual son desplazados en la orientación y educación de sus hijos, trayendo como consecuencia la adopción de comportamientos descontextualizados con su origen.

5. Es importante proponer alternativas para resolver los problemas de la familia contemporánea; pero se considera de mayor trascendencia la ejecución de programas de prevención y promoción de la salud mental dirigidos a adolescentes y jóvenes, que en el futuro serán padres de familia, para que no transmitan a su descendencia las conductas disfuncionales de algunos jóvenes en la actualidad.

\section{REFERENCIAS BIBLIOGRAFICAS}

AMERICAN PSYCHIATRIC Association, Manual Diagnóstico y Estadístico de los Trastornos Mentales DSM IV, Manual Moderno.

BuendíA, J. (1996). Psicopatología en niños y adolescentes. Desarrollo actuales. Madrid: Pirámide.

BARAVALLE G. y VACARREZA L., (1998), Anorexia. Teoría y clínica psicoanalítica, $2^{\circ}$. reimpresión, Buenos Aires, Paidós, p. 57.

BRUCH, H. 1971. Family transactions in eating disorders. Comprehensive Psychiatry Vol.12 $\mathrm{N}^{\circ} 3$ : 238-248.

CAPARros N. y SANFEliÚ I., (1997), La anorexia, una locura del cuerpo, Madrid, Biblioteca Nueva, p. 21.

GÁFARo, AlejANDRA. (2001). Anorexia y bulimia, todo lo que necesitas saber sobre los trastornos de la alimentación. Editorial Norma. Bogotá-Colombia.

GómEZ, G. 1993 Detección de anomalías de la conducta alimentaria en estudiantes universitarios: obesidad, bulimia y anorexia nerviosa. Revista Mexicana de Psicología Vol. 10: 17-26.

GómEZ G. Variables cognoscitivas y actitudinales asociadas con imagen corporal y desordenes del comer: problemas de peso. InvPsc; 1994;3(1):95-112.

Lugli Z., ViVAS E., (2001), “Trastornos de alimentación y control personal de la conducta”, Salud Pública de México, p. 14, Cuernavaca Méx., vol. 43.

MÉNDEZ A. CARLOS E. (1995). Metodología diseño y desarrollo del proceso de investigación. Bogotá, Colombia.

Minuchin, S., B.L. Rosman y L. BAKER 1978. Psychosomatics families: anorexia nervosa in context. Cambridge: Harvard University. 371 pp.

RAuSCH C., BAY L., (1997), Anorexia nerviosa y bulimia, Buenos Aires, $4^{\text {a }}$. Reimpresión, Paidós, p. 31.

Rodríguez AM, Martínez JM, Novalbos JP, Ruiz MA, JimÉnez D. Ejercicio físico y hábitos alimentarios: un estudio en adolescentes de Cádiz. RevEsp S Public 2003;6:1-6. 
RodrígueZ SACRISTÁn, J.(1995). Psicopatología del Niño y del Adolescente. Sevilla: SPUG.

SALDAÑA C. 2000 Consecuencias psicopatológicas del seguimiento de dietas. Formación continuada en Nutrición y Obesidad. Vol 3: 2812-2889.

UniKel C, MoRa J, Gómez G. (1999) Percepción de la gordura en adolescentes y su relación con las conductas anómalas del comer. Int J Psic 1999; 33(1):11-29.

UNIKEL C, GómEZ G. (1999) Espectro de los trastornos de la conducta alimentaria: grupo normal, con alto riesgo y clínico. RevMexPsc 1999; 16(1): 141-151.

WiCks-Nelson, R., e IsRael, A.C. (1997). Psicopatología del Niño y del Adolescente. Madrid: Prentice Hall.

\section{Linkografía}

http://www.dmedicina.com/salud/psiquiatricas/anorexia.html 2008. Madrid. Unidad Editorial, Revistas. (noviembre, 2012).

Álvarez, Geraldine y Meni, María. (s/f). Bulimia y Anorexia. http://www.monografias.com/trabajos/ buliano/buliano.shtml.

Cook-Darzens et al., (2005). www.bulimia.com y www.eatingdisordersblogs.com (noviembre, 2012).

Edelstein, (1999) http://www.rieoei.org/deloslectores/343Zagalaz.pdf

Garfinkel y Garner, (2012). http://comunidad-escolar.pntic.mec.es/668/tribuna.html (noviembre, 2012).

Morduchowicz, Marcon, Sylvestre y Ballestrini, (2010) p. 6. http://www.dmedicina.com/salud/ psiquiatricas/bulimia.html 2008. Madrid. Unidad Editorial, Revistas. (noviembre, 2012).

Strober y Yager (2012). http://geosalud.com/Nutricion/anorexia_bulimia.htm (noviembre, 2012).

White (2011). http://www.tuotromedico.com/temas/obesidad.htm Con el patrocinio de SANITAS. (noviembre, 2012).

www.lawebempresa20.com 